\title{
Modified multistep model predictive control for three-phase induction motor drive system considering the common-mode voltage minimization
}

\author{
Bao Binh Pho', Nguyen Van $\mathrm{Cao}^{2}$, Tran Minh Hoan ${ }^{3}$, Phuong $\mathrm{Vu}^{4}$ \\ ${ }^{1-4}$ School of Electrical Engineering, Hanoi University of Science and Technology, Ha Noi, Vietnam \\ ${ }^{1}$ National University of Civil Engineering, Ha Noi, Vietnam
}

\begin{tabular}{|c|c|}
\hline Article Info & ABSTRACT \\
\hline Article history: & It is acknowledged that the common-mode voltage may have detrimental \\
\hline Received Jun 12, 2021 & effects on an induction motor (IM) drive system if not properly addressed. \\
\hline Revised Sep 13, 2021 & method for IM drive system considering the common-mode voltage \\
\hline Accepted Sep 20, 2021 & $\begin{array}{l}\text { minimization is proposed. This research uses a multi-objective cost function, } \\
\text { before applying the Sphere Decoding Algorithm to find the optimal control }\end{array}$ \\
\hline Keywords: & $\begin{array}{l}\text { input. The results show that the proposed control method not only reduces } \\
\text { the common-mode voltage significantly but also mitigates the computational }\end{array}$ \\
\hline Cascaded H-bridge inveter & burden of the microprocessor without affecting the system performance. The \\
\hline Common-mode voltage & proposed control method is simulated by MATLAB/Simulink for an IM \\
\hline Field oriented control & \\
\hline
\end{tabular}

Multistep predictive current

control

This is an open access article under the CC BY-SA license.

Sphere decoder

Corresponding Author:

Phuong Vu

School of Electrical Engineering

Hanoi University of Science and Technology

No.1, Dai Co Viet Road, Hai Ba Trung, Hanoi, Vietnam

Email: phuong.vuhoang@hust.edu.vn

\section{INTRODUCTION}

Recently, multilevel inverters (MLI) are widely used in high-power industrial applications because of these advantages: low output voltage distortion, reducing the voltage level on semiconductor devices and lowering the du/dt changing speed [1]. Especially, in comparison with other MLI topologies such as neutralpoint clamped [2], flying capacitors [3], multi-modular converters (MMC), the cascaded h-bridge (CHB) [4][6] has emerged as a prominent one for driving IM system due to its high degree of modularity, allowing upgrade and replacement easily. Furthermore, for the applications of induction motor drives, the connection of CHB-MLI with multi-phase rectifier transformer ensures the current drawn from the grid have a sinusoidal waveform, decreases the total harmonic distortion injected into the grid.

On the other hand, a considerable drawback of CHB-MLI that produces the common-mode voltage (CMV) between the neutral points of the load and the converter [7], which can be calculated by (1) and illustrated by Figure 1. Due to this CMV, high-frequency electromagnetic interference (EMI) is introduced, causing problems regarding measurement and system monitoring [8]. Furthermore, in IM driving system, the CMV generates the common-mode current making detrimental effects on the motor's productivity and endurance [9]. There have been many undergoing researches to minimize the CMV, for instance the addition of the EMI active or passive filter [10], [11]. However, this hardware solution can lead to an increase in the 
size and cost of the system. Therefore, mitigation of CMV based on control method approaches like SVM [12], [13], MPC [14], [15] is preferred.

$$
v_{Z N}=v_{C M V}=\frac{1}{3}\left(v_{A N}+v_{B N}+v_{C N}\right)
$$

Among these control methods, multistep MPC has significant benefits since it takes the switching nature of the power converter. It is also shown that multistep MPC can improve the steady-state performance of the system better than single-step MPC [16]-[20]. However, in [21] multistep model predictive control (MPC) with CMV minimization target applies to RL load as the case study only. Regarding IM driving system case study, in [22], the multistep MPC controller does not consider CMV minimization target since it is designed toward optimizing the switching effort.

Therefore, this paper proposes a modified multistep MPC compared to [22]. To account for CMV, an additional term that penalizes the CMV is added to the cost function before using the SDA algorithm to solve the optimization problem. As evidence from the simulation results, this approach also cuts down the computational burden, confirms the feasibility of this proposal in practical adoption.

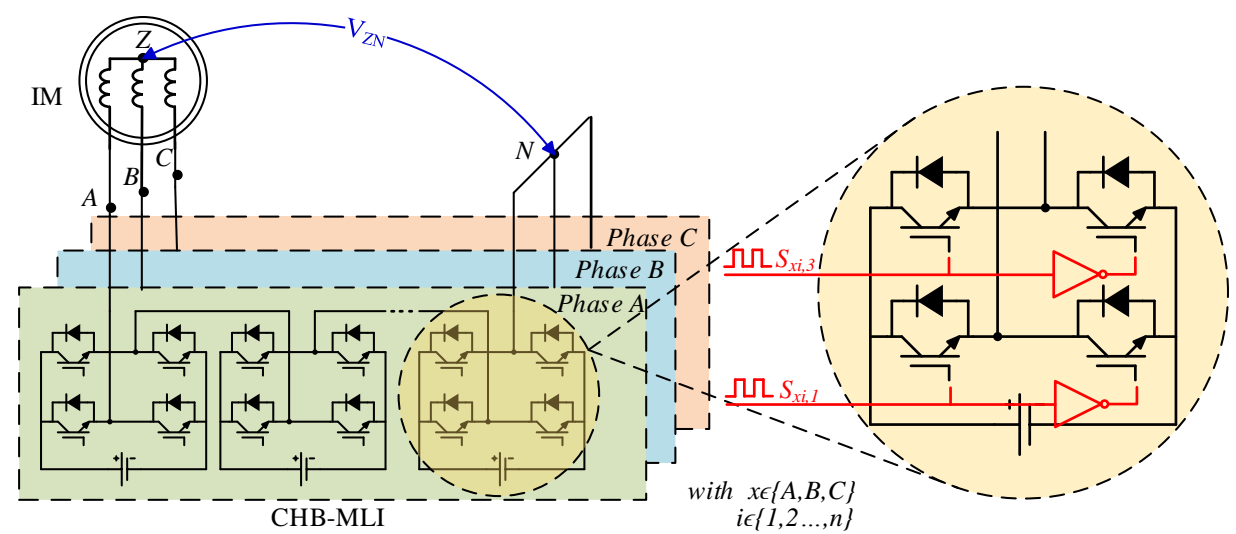

Figure 1. IM drive system with CHB-MLI.

\section{MULTISTEP MODEL PRECDICTIVE CONTROL METHOD}

In general, the proposed control method for IM drive system follows the field oriented controller (FOC) approach, as depicted in Figure 2 [23]-[25]. There are two major control loops. The classical PI controller takes care of the outer speed and flux control loop, whereas the inner current loop is controlled by the multistep MPC. Notice that two different weighting factors which account for CMV minimization and reduce switching effort are applied to the cost function.

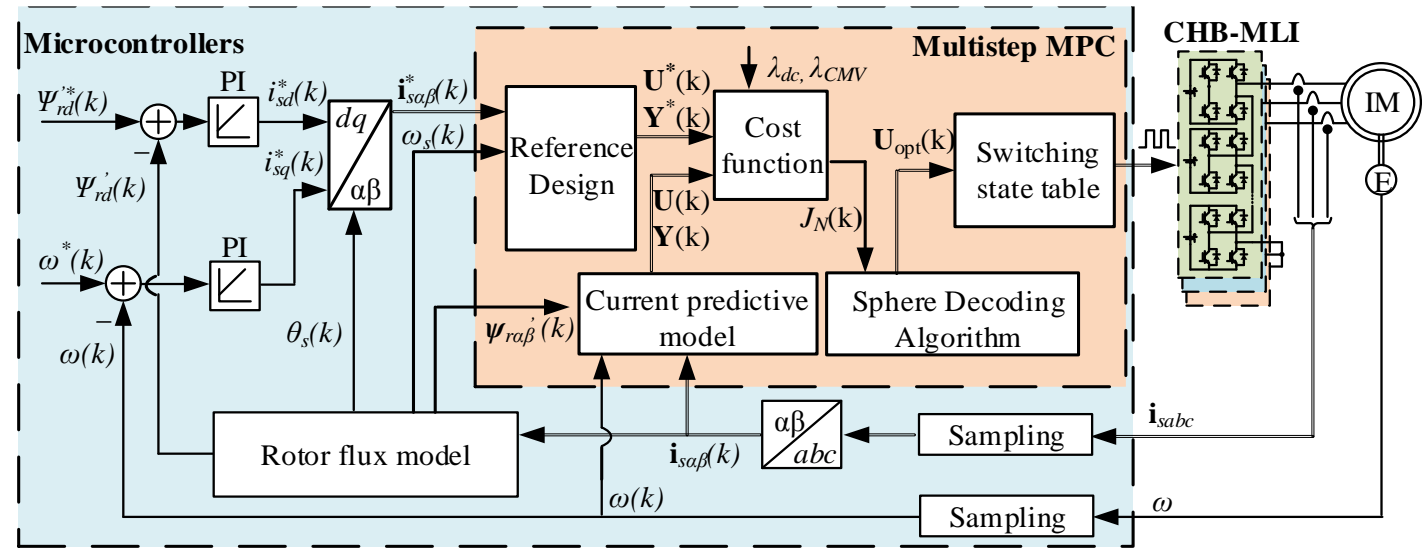

Figure 2. The control scheme for induction motor with inner multistep MPC current control loop 


\subsection{Current predictive model}

First, the physical model of the IM drive system is formulated as differential equations, representing the relationship between system parameters. After that, a state-space representation of the system can be obtained. The state-space representation of the continuous-time prediction model of the IM drive system powered by a CHB-MLI can be written as (2):

$$
\left\{\begin{array}{l}
d \boldsymbol{x}(t)=\boldsymbol{A}^{\prime}(t) \cdot \boldsymbol{x}(t)+\boldsymbol{B}^{\prime} \boldsymbol{u}(t) \\
\boldsymbol{y}(t+1)=\boldsymbol{C} \cdot \boldsymbol{x}(t+1)
\end{array}\right.
$$

With

$$
\begin{aligned}
& \boldsymbol{A}^{\prime}(t)=\left[\begin{array}{cccc}
-\left(\frac{1}{\sigma T_{s}}+\frac{1-\sigma}{\sigma T_{r}}\right) & 0 & \frac{1-\sigma}{\sigma T_{r}} & \frac{1-\sigma}{\sigma} \omega(t) \\
0 & -\left(\frac{1}{\sigma T_{s}}+\frac{1-\sigma}{\sigma T_{r}}\right) & -\frac{1-\sigma}{\sigma} \omega(t) & \frac{1-\sigma}{\sigma T_{r}} \\
\frac{1}{T_{r}} & 0 & -\frac{1}{T_{r}} & -\omega(t) \\
0 & \frac{1}{T_{r}} & \omega(t) & \frac{-1}{T_{r}}
\end{array}\right] ; \\
& \boldsymbol{B}^{\prime}=\frac{V_{d c}}{3 \sigma L_{s}}\left[\begin{array}{ccc}
2 & -1 & -1 \\
0 & \sqrt{3} & -\sqrt{3} \\
0 & 0 & 0 \\
0 & 0 & 0
\end{array}\right] \\
& \boldsymbol{C}=\left[\begin{array}{llll}
1 & 0 & 0 & 0 \\
0 & 1 & 0 & 0
\end{array}\right]
\end{aligned}
$$

Where the state vector $\boldsymbol{x}=\left[\begin{array}{llll}i_{s \alpha} & i_{s \beta} & \psi_{r \alpha}^{\prime} & \psi_{r \beta}^{\prime}\end{array}\right]^{T} \in \square^{4}$, the control input vector $\boldsymbol{u}=\boldsymbol{S}=$ $\left[\begin{array}{lll}S_{A} & S_{B} & S_{C}\end{array}\right]^{T} \in \mathbb{U}$ (the set $\mathrm{U}$ is the set of voltage level combinations in three phase), output vector $\boldsymbol{y}=$ $\boldsymbol{i}_{s \alpha \beta}=\left[\begin{array}{ll}i_{s \alpha} & i_{s \beta}\end{array}\right]^{T} \in \square^{2}$, notice that $\boldsymbol{A}^{\prime}(t)$ is a time-variant matrix because it consists of time-variant variables $\omega(t)$. Specifically, $i_{s \alpha}, i_{s \beta}, \psi_{r \alpha}^{\prime}, \psi_{r \beta}^{\prime}$ stand for stator currents and rotor flux linkages in the stationary $\alpha \beta$-framework. System parameters $\sigma, T_{s}, T_{r}$ and $\omega$ are defined as total leakage factor, stator time constant, rotor time constant and rotor speed respectively. After applying the Forward-Euler discretization with a sampling period of $T$, the discrete-time prediction model can be expressed as (3).

$$
\left\{\begin{array} { l } 
{ x ( k + 1 ) = A \cdot x ( k ) + B u ( k ) } \\
{ y ( k + 1 ) = C \cdot x ( k + 1 ) }
\end{array} \text { with } \left\{\begin{array}{l}
A=I_{4}+T \cdot A^{\prime} \in \square^{4 \times 4} \\
B=T \cdot B^{\prime} \in \square^{4 \times 3}
\end{array}\right.\right.
$$

Based on the prediction model in (4), the control input and output sequence over the prediction horizon $\mathrm{N}>1$ can be constructed as (5) with relationships in (6).

$$
\begin{aligned}
& \mathbf{U}(\mathrm{k})=\left[\begin{array}{llll}
(\mathbf{u}(k))^{T} & (\mathbf{u}(k+1))^{T} & \ldots & (\mathbf{u}(k+N-1))^{T}
\end{array}\right]^{T} \in \mathrm{U}^{N} \\
& \mathbf{Y}(\mathrm{k})=\left[\begin{array}{llll}
(\mathbf{y}(k+1))^{T} & (\mathbf{y}(k+2))^{T} & \ldots & (\mathbf{y}(k+N))^{T}
\end{array}\right]^{T} \in \square^{2 N} \\
& Y(k)=\Gamma x(k)+Y U(k) \text { Where } \Gamma=\left[\begin{array}{c}
C A \\
C A^{2} \\
\vdots \\
C A^{N}
\end{array}\right], Y=\left[\begin{array}{ccccc}
C B & 0 & \cdots & 0 & 0 \\
C A B & C B & \cdots & 0 & 0 \\
\vdots & \vdots & \ddots & \vdots & \vdots \\
C A^{N-1} B & C A^{N-2} B & \cdots & C A B & C B
\end{array}\right]
\end{aligned}
$$

\subsection{Reference design}

Because the stator current and rotor flux signals are slower than electrical ones, so that over the finite prediction horizon $\mathrm{N}$, these parameters are assumed to be constant. The output reference sequence for the current controller is denoted by (7).

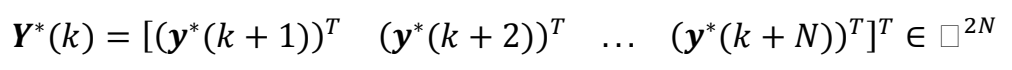


$\operatorname{Where}^{*}(\ell)=\left[\sqrt{i_{s \alpha}^{2}(k)+i_{s \beta}^{2}(k)} \cdot \sin \left(\omega_{s} \ell T+\varphi_{0}\right) \quad \sqrt{i_{s \alpha}^{2}(k)+i_{s \beta}^{2}(k)} \cdot \sin \left(\omega_{s} \ell T+\varphi_{0}+\frac{\pi}{2}\right)\right]^{T} \ell \in$

$\{k+1 ; \ldots ; k+N\}, \varphi_{0}=\arctan \left(\frac{i_{s \beta}(k)}{i_{s \alpha}(k)}\right)$ is the initial angle between $i_{s \alpha}$ and $i_{s \beta}$.

The control input reference sequence $(8)$ is designed while considering a null CMV $\left(v_{C M V}=0\right)$.

$$
\begin{aligned}
& \boldsymbol{U}^{*}(k)=\left[\begin{array}{llll}
\left(\boldsymbol{u}^{*}(k)\right)^{T} & \left(\boldsymbol{u}^{*}(k+1)\right)^{T} & \ldots & \left(\boldsymbol{u}^{*}(k+N-1)\right)^{T}
\end{array}\right]^{T} \\
& \text { Where } \boldsymbol{u}^{*}(\ell)=\frac{\sigma L_{S}}{V_{d c}}\left[\begin{array}{cc}
1 & 0 \\
\frac{-1}{2} & \frac{\sqrt{3}}{2} \\
\frac{3}{2} & \frac{-\sqrt{3}}{2}
\end{array}\right] \cdot\left(\frac{d \boldsymbol{i}_{s \alpha \beta}^{*}(\ell)}{d t}+\left(\frac{1}{\sigma T_{s}}+\frac{1-\sigma}{\sigma T_{r}}\right) \boldsymbol{i}_{s \alpha \beta}^{*}(\ell)-\left[\begin{array}{cc}
\frac{1-\sigma}{\sigma T_{r}} & \frac{1-\sigma}{\sigma} \omega \\
-\frac{1-\sigma}{\sigma} \omega & \frac{1-\sigma}{\sigma T_{r}}
\end{array}\right] \boldsymbol{\psi}_{r \alpha \beta}^{\prime}(k)\right)
\end{aligned}
$$

\subsection{Cost function}

To achieve the desired CMV minimization target, the cost function (9) is modified match up to [22]. In addition to output reference tracking, the proposed method which is designed to minimize the CMV also tracks the input references.

$$
J_{N}(k)=\sum_{l=k}^{k+N-1}\left(\left\|\boldsymbol{y}(\ell+1)-\boldsymbol{y}^{*}(\ell+1)\right\|_{2}^{2}+\lambda_{d c}\|\Delta \boldsymbol{u}(\ell)\|_{2}^{2}+\lambda_{C M V}\left\|\boldsymbol{u}(\ell)-\boldsymbol{u}^{*}(\ell)\right\|_{2}^{2}\right)
$$

In $(9), \boldsymbol{u}(\ell)$ is the candidate control-input that generates the output current prediction $\boldsymbol{y}(\ell+1)=$ $\boldsymbol{i}_{\alpha \beta}(\ell+1) ; \Delta u(\ell)=u(\ell)-u(\ell-1) ; \lambda_{d c}$ is the weighting factor for lessening switching effort, $\lambda_{C M V}$ is the weighting factor for CMV minimization. Representation of (9) in matrix form can be written as (10):

$$
\begin{aligned}
& J_{N}(k)=\left\|\boldsymbol{Y}(k)-\boldsymbol{Y}^{*}(k)\right\|_{2}^{2}+\lambda_{d c}\|\boldsymbol{S} \boldsymbol{U}(k)-\boldsymbol{E} \boldsymbol{u}(k-1)\|_{2}^{2}+\lambda_{C M V}\left\|\boldsymbol{U}(k)-\boldsymbol{U}^{*}(k)\right\|_{2}^{2} \\
& \text { Where } \boldsymbol{S}=\left[\begin{array}{cccc}
\boldsymbol{I}_{3} & \mathbf{0}_{3} & \cdots & \mathbf{0}_{3} \\
-\boldsymbol{I}_{3} & \boldsymbol{I}_{3} & \cdots & \mathbf{0}_{3} \\
\mathbf{0}_{3} & -\boldsymbol{I}_{3} & \cdots & \mathbf{0}_{3} \\
\vdots & \vdots & \ddots & \mathbf{0}_{3} \\
\mathbf{0}_{3} & \mathbf{0}_{3} & \cdots & \boldsymbol{I}_{3}
\end{array}\right]_{[3 N x 3 N]}, \boldsymbol{E}=\left[\begin{array}{c}
\boldsymbol{I}_{3} \\
\mathbf{0}_{3} \\
\vdots \\
\mathbf{0}_{3}
\end{array}\right]_{[3 N x 3]}
\end{aligned}
$$

\subsection{Sphere decoding algorithm (SDA)}

In this job, the SDA is adopted to solve the modified optimization problem, to obtain the input sequence $\boldsymbol{U}(k)=\boldsymbol{U}_{\text {opt }}(k)$ that minimizes the cost function $J_{N}(k)$ in detail. The computational process will be presented concisely. Further detail about the SDA can be found in [18], [19]. By using (5), the cost function (10) becomes:

$$
J_{N}(k)=\boldsymbol{U}(k)^{T} \boldsymbol{W} \boldsymbol{U}(k)+2 \mathbf{F}(k)^{T} \boldsymbol{U}(k)+\boldsymbol{\varepsilon}(k)
$$

Where

$$
\begin{aligned}
& \boldsymbol{W}=Y^{T} Y+\lambda_{d c} \boldsymbol{S}^{T} \boldsymbol{S}+\lambda_{C M V} \boldsymbol{I}_{3 N} \\
& \boldsymbol{F}(k)=Y^{T} \Gamma \boldsymbol{x}(k)-Y^{T} \boldsymbol{Y}^{*}(k)-\lambda_{d c} \boldsymbol{S}^{T} \boldsymbol{E} \boldsymbol{u}(k-1)-\lambda_{C M V} \boldsymbol{U}^{*}(k) \\
& \boldsymbol{\varepsilon}(k)=\left[\left\|\Gamma \boldsymbol{x}(k)-\boldsymbol{Y}^{*}(k)\right\|_{2}^{2}+\lambda_{d c}\|\boldsymbol{E} \boldsymbol{u}(k-1)\|_{2}^{2}+\lambda_{C M V}\left\|\boldsymbol{U}^{*}(k)\right\|_{2}^{2}\right]
\end{aligned}
$$

By solving $\frac{\partial J_{N}}{\partial \boldsymbol{U}}=0$, the optimal solution $\boldsymbol{U}_{u c}$ is yielded in (12), which does not necessary belong to the finite control-input set $\mathrm{U}$.

$$
\boldsymbol{U}_{u c}=-\boldsymbol{W}^{-1} \boldsymbol{F}(k)
$$

Because $\mathbf{W}$ is a symmetric and positive definite matrix for $\lambda_{d c}, \lambda_{C M V}>0$, there exists only one unique invertible lower triangular matrix $H$, which can be obtained by performing the Cholesky decomposition to $W^{-1}: W^{-1}=\boldsymbol{H}^{-1} \boldsymbol{H}^{-T}$. Hence, the matrix $\boldsymbol{H}$ will satisfy the equation: $W=\boldsymbol{H}^{T} \boldsymbol{H}$. Consequently, the cost function (11) can be rewritten as (13). Figure 3 illustrates the graphical representation of a MPC problem in two-dimensional space. To simplify, control input vectors referring to the same ellipse will have the same cost value. By transforming the cost function (11) into (13), the original ellipses are transformed into circles. This procedure plays an important role in SDA. 


$$
J_{N}(k)=\left\|\boldsymbol{H} \boldsymbol{U}(k)-\overline{\boldsymbol{U}}_{u c}(k)\right\|_{2}^{2} \text { where } \overline{\mathbf{U}}_{u c}(k)=\mathbf{H} \mathbf{U}_{u c}(k)
$$

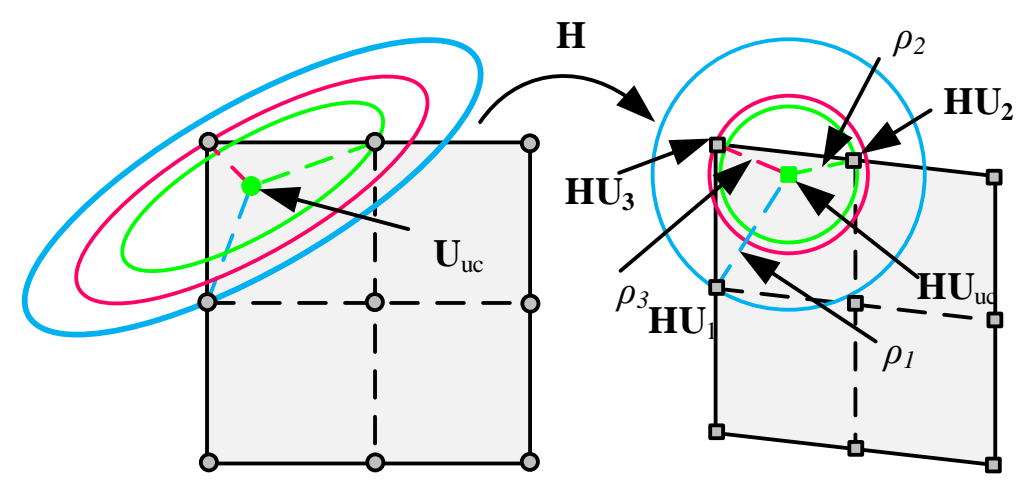

Figure 3. Representation of the meaning of the matrix $\mathrm{H}$

The optimal control input for prediction horizon $\mathrm{N}$ can be presented as (14):

$$
\boldsymbol{U}_{\text {opt }}(k)=\left[\begin{array}{llll}
\boldsymbol{u}_{a b c}(k) & \boldsymbol{u}_{a b c}(k+1) & \ldots & \boldsymbol{u}_{a b c}(k+N-1)
\end{array}\right]^{T}
$$

Now, reconstruct (14) into a sequence (element by element) as (15):

$$
\boldsymbol{U}_{\text {opt }}=\left[\begin{array}{llll}
u_{1} & u_{2} & \ldots & u_{3 N}
\end{array}\right]^{T} \text { với } i \in\{1,2, \ldots, 3 N\}
$$

Figure 4 depicts the evaluation process of each element $u_{i}$, so-called a node, of the control input sequence U in (15). Figure 5 illustrates the flow diagram of SDA. First, an initial sphere $S_{i n i}$ is defined with an initial center $\overline{\boldsymbol{U}}_{u c}(k)$ calculated by (16) and a selective initial radius $p_{i n i}$. This initial sphere $S_{i n i}$ should be small enough to contain at least one solution $\boldsymbol{U}(k)$. Then during the evaluation process, the size of the sphere is decreased until there is only one solution contained in it, and that is the optimal control input sequence. Each time a node is visited, the radius $p_{i}$ is calculated by (17) before evaluating the condition (18). If $p_{i}$ violates condition (18), the node $u_{i}$ will be discarded and its following nodes (from $u_{i+1}$ to $u_{3 N}$ ) will be discarded without performing any computation to avoid unnecessary computation. The control input having the smallest radius will be the optimal solution $\boldsymbol{U}(k)=\boldsymbol{U}_{\text {opt }}(k)$.

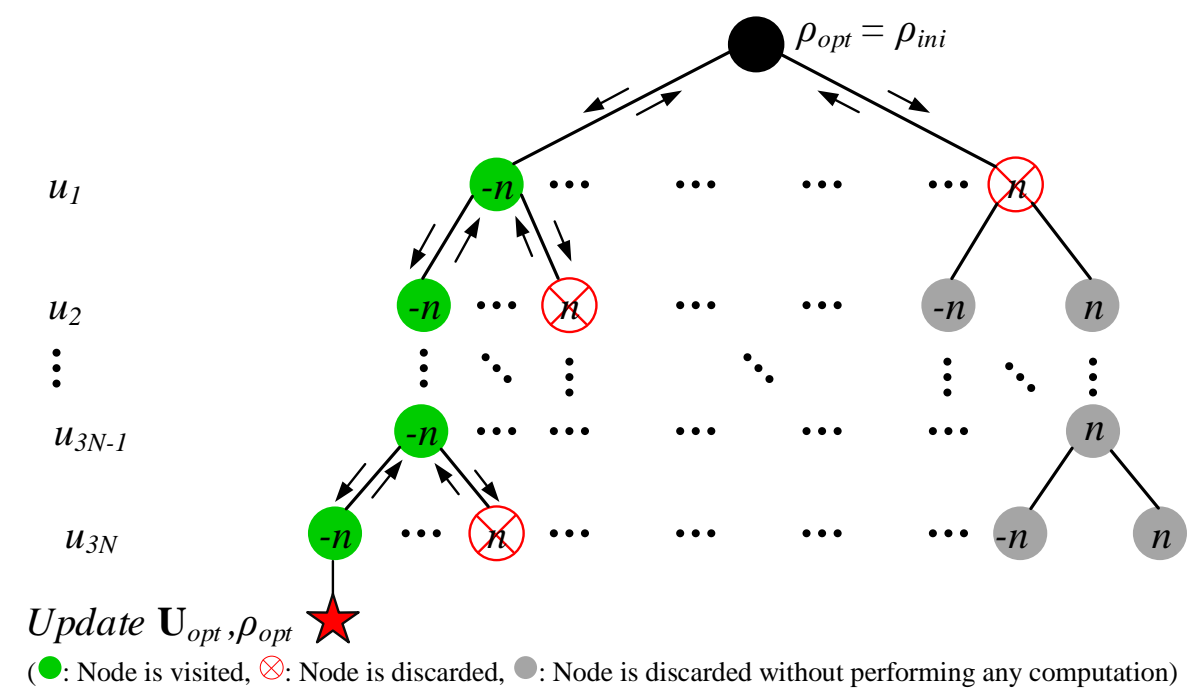

Figure 4. A tree-diagram of the control input sequence 


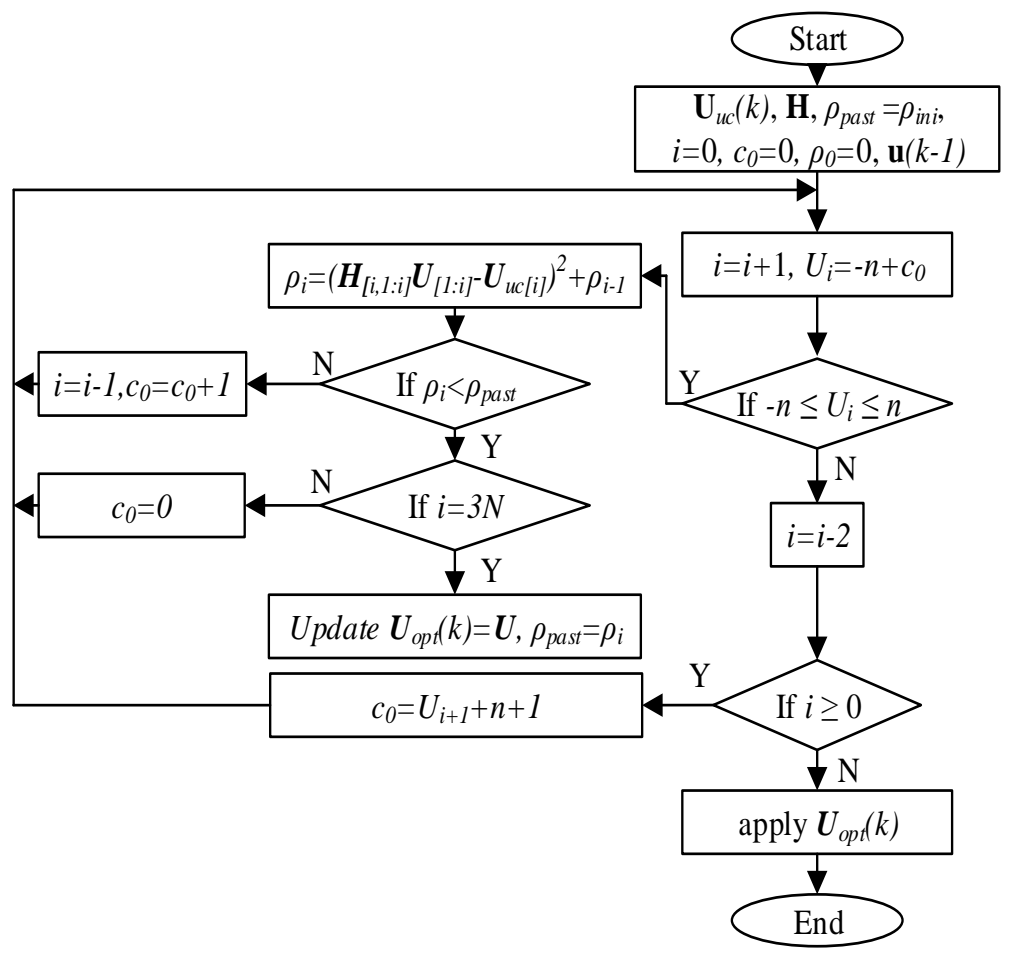

Figure 5. SDA flow diagram [20]

$$
\begin{aligned}
& \bar{U}_{u c}(k)=H\left(-W^{-1}\right) F(k) \\
& \rho_{i}^{2}=\|\underbrace{\left[h_{i 1} \ldots h_{i i}\right]}_{H_{[i, 1 i]}} \underbrace{\left[u_{1} \ldots u_{i}\right]^{T}}_{U_{o p t[1: i]}}-\underbrace{\bar{u}_{u c, i}}_{\tilde{U}_{u c[i]}}\|_{2}^{2}+\rho_{i-1}^{2}
\end{aligned}
$$

Condition: $\rho_{i}^{2} \leq \rho_{o p t}^{2}$

\subsection{Switching state table}

When the optimal solution $\boldsymbol{U}(\boldsymbol{k})$ is obtained, only the first element $\boldsymbol{u}_{\boldsymbol{a b c}}(\boldsymbol{k})$ is employed to the find the switching states for the inverter. Notice that $\boldsymbol{u}_{\boldsymbol{a b c}}(\boldsymbol{k})$ is the phase voltage levels. Table 1 shows the switching states selection strategy clearly. In Table 1, variable represent the phases, the index notation denotes the position-number of a $\mathrm{H}$-bridge in phase, is the voltage level of phase, is the voltage level of the $\mathrm{H}$-bridge in phase, are the switching states of valve 1 and 3 of the H-bridge in phase. Figure 1 illustrates this switching state selection strategy.

\begin{tabular}{|c|c|c|c|c|c|}
\hline$S_{x}$ & $S_{x 1}\left(S_{x 1,1} ; S_{x 1,3}\right)$ & $S_{x 2}\left(S_{x 2,1} ; S_{x 2,3}\right)$ & $\begin{array}{c}S_{x i}\left(S_{x i, 1} ; S_{x i, 3}\right) \\
S_{x 3}\left(S_{x 3,1} ; S_{x 3,3}\right)\end{array}$ & $S_{x 4}\left(S_{x 4,1} ; S_{x 4,3}\right)$ & $S_{x 5}\left(S_{x 5,1} ; S_{x 5,3}\right)$ \\
\hline+5 & $1(1 ; 0)$ & $1(1 ; 0)$ & $1(1 ; 0)$ & $1(1 ; 0)$ & $1(1 ; 0)$ \\
\hline+4 & $1(1 ; 0)$ & $1(1 ; 0)$ & $1(1 ; 0)$ & $1(1 ; 0)$ & $0(0 ; 0)$ \\
\hline+3 & $1(1 ; 0)$ & $1(1 ; 0)$ & $1(1 ; 0)$ & $0(0 ; 0)$ & $0(0 ; 0)$ \\
\hline+2 & $1(1 ; 0)$ & $1(1 ; 0)$ & $0(0 ; 0)$ & $0(0 ; 0)$ & $0(0 ; 0)$ \\
\hline+1 & $1(1 ; 0)$ & $0(0 ; 0)$ & $0(0 ; 0)$ & $0(0 ; 0)$ & $0(0 ; 0)$ \\
\hline 0 & $0(0 ; 0)$ & $0(0 ; 0)$ & $0(0 ; 0)$ & $0(0 ; 0)$ & $0(0 ; 0)$ \\
\hline-1 & $0(0 ; 0)$ & $0(0 ; 0)$ & $0(0 ; 0)$ & $0(0 ; 0)$ & $-1(0 ; 1)$ \\
\hline-2 & $0(0 ; 0)$ & $0(0 ; 0)$ & $0(0 ; 0)$ & $-1(0 ; 1)$ & $-1(0 ; 1)$ \\
\hline-3 & $0(0 ; 0)$ & $0(0 ; 0)$ & $-1(0 ; 1)$ & $-1(0 ; 1)$ & $-1(0 ; 1)$ \\
\hline-4 & $0(0 ; 0)$ & $-1(0 ; 1)$ & $-1(0 ; 1)$ & $-1(0 ; 1)$ & $-1(0 ; 1)$ \\
\hline-5 & $-1(0 ; 1)$ & $-1(0 ; 1)$ & $-1(0 ; 1)$ & $-1(0 ; 1)$ & $-1(0 ; 1)$ \\
\hline
\end{tabular}

Table 1. Switching state selection strategy 


\section{SIMULATION RESULTS}

The proposed control scheme is simulated by MATLAB/Simulink for IM drive system with an 11level CHB, DC voltage supply per CHB $V_{d c}=600 \mathrm{~V}$ and the prediction horizon $\mathrm{N}=3$. The IM drive system parameters and the simulation scenarios are shown in Tables 2 and 3 respectively. To definitely demonstrate the benefits of the proposed control method in company with [22], the CMV weighting factors are applied to the cost function from $1,35 \mathrm{~s}$ to $1,45 \mathrm{~s}$. The sampling period $T=50 \mu \mathrm{s}$.

Table 2. IM drive system parameters

\begin{tabular}{ccc}
\hline Nominal Power & $P_{d m}$ & $1119 \mathrm{KW}$ \\
\hline Nominal Electromagnetic Torque & $M_{d m}$ & $6906 \mathrm{Nm}$ \\
Nominal Current & $I_{d m}$ & $234.18 \mathrm{~A}$ \\
Nominal Voltage & $U_{d m}$ & $3.3 \mathrm{kV}$ \\
Nominal Speed & $\omega_{d m}$ & $1470 \mathrm{rpm}$ \\
\hline
\end{tabular}

Table 3. Simulation scenarios

\begin{tabular}{|c|c|c|c|c|c|}
\hline Time & $0-0.5[\mathrm{~s}]$ & $0.5-1[\mathrm{~s}]$ & $1-1.25[\mathrm{~s}]$ & $1.25-1.35[\mathrm{~s}]$ & $1.35-1.45[\mathrm{~s}]$ \\
\hline Reference speed & $\begin{array}{c}\text { Magnetization } \\
\omega_{\text {ref }}=0\end{array}$ & $\begin{array}{c}\text { Gradually increase speed } \\
\omega_{\text {ref }}=1470 \mathrm{rpm}\end{array}$ & \multicolumn{3}{|c|}{ Speed Stabilization $\omega_{\text {ref }}=1470 \mathrm{rpm}$} \\
\hline Weighting Factors & & $\lambda_{d c} \approx 0 ; \lambda_{C M V} \approx 0$ & & ${ }_{C M V} \approx 0 \lambda_{d c}=$ & $\lambda_{C M V}=4 \lambda_{d c}=4$ \\
\hline
\end{tabular}

It is presented in Figure 6 that the rotor flux, the rotor speed, and the electromagnetic torque are smoothly ramped-up and quickly reach their reference values. Figure 7 illustrates the stator current and the CMV at the steady-state. The effectiveness of the proposed control method is clearly demonstrated when the CMV weighting factor is applied in the period of (1.35-1.45) [s]. Especially in (1.15-1.25) [s] and (1.25-1.35) [s], the CMV is registered at $1800 \mathrm{~V}\left(3 V_{d c}\right)$, while in $(1.35-1.45)$ [s], the CMV is drastically reduced to $200 \mathrm{~V}$ $\left(\frac{1}{3} \cdot V_{d c}\right)$. Moreover, Table 4 depicts the number of evaluated nodes during the SDA optimization process.

It can be seen that this number is significantly decreased from 144,969 nodes to 88,396 nodes, indicates the reduction of the computational burden evidently. However, these benefits are traded for a small increase of THD and switching effort, specifically from $0.58 \%$ to $0.61 \%$ for THD and from 2.43 to 2.78 times per cycle for switching effort. Finally, Figure 8 shows that the stator current in $\alpha \beta$-framework is attained its reference for all simulation scenarios with the error below 5\%. Hence, the additional CMV minimization target does not affect the system performance.

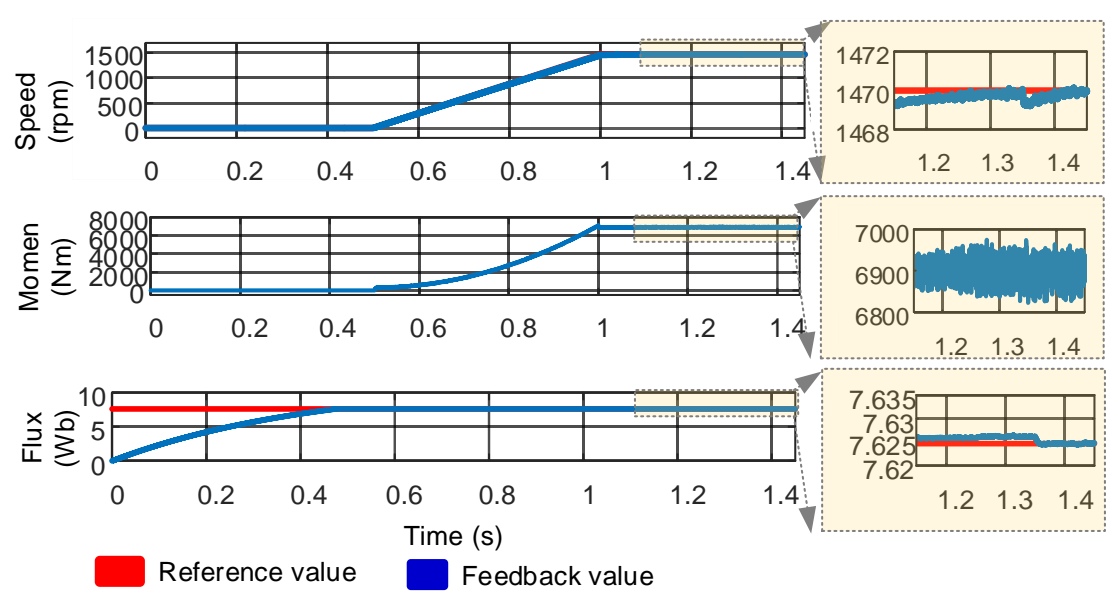

Figure 6. Graphs of motor speed, torque and motor flux 


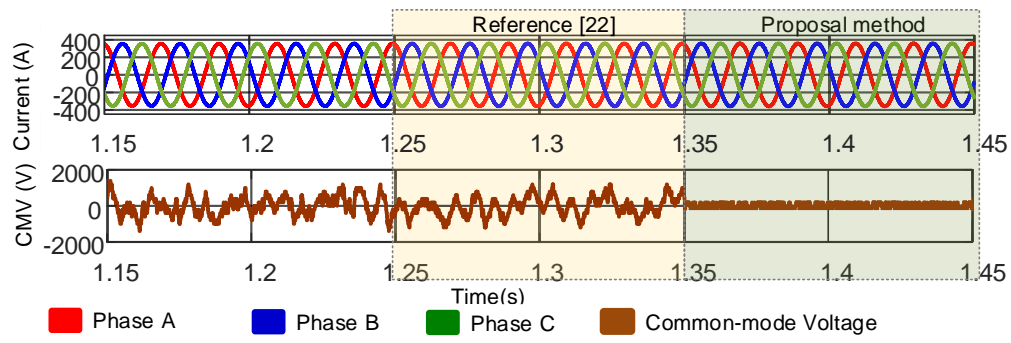

Figure 7. Graphs of stator currents and common-mode voltage

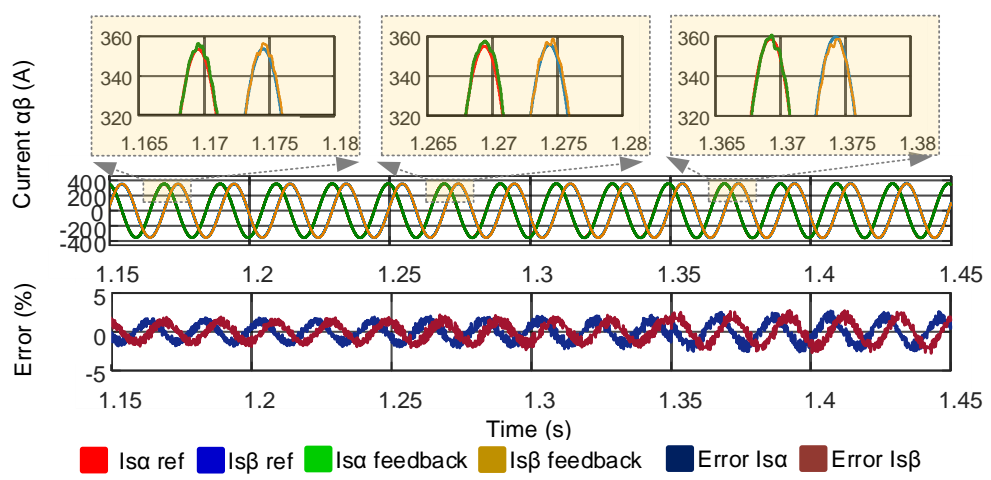

Figure 8. Graph of stator current and error value in the $\alpha \beta$ - framework

Table 4. Results about THD, switching effort, and number of evaluated nodes

\begin{tabular}{cccc}
\hline Time & $1.15-1.25[\mathrm{~s}]$ & $1.25-1.35[\mathrm{~s}]$ ( Reference [22]) & $1.35-1.45[\mathrm{~s}]$ (Proposal) \\
\hline THD & $0.46 \%$ & $0.58 \%$ & $0.61 \%$ \\
Switching Effort & 3.26 (times/ cycle) & 2.43 (times/ cycle) & 2.78 (times/ cycle) \\
Number of evaluated nodes & 314336 (nodes) & 144969 (nodes) & 88396 (nodes) \\
\hline
\end{tabular}

\section{CONCLUSION}

This paper proposes a modified multistep MPC for IM drive system with a CHB-MLI. This proposed method achieves two main contributions: firstly, the reduction of CMV while retaining the current quality and switching effort at the desired range. Secondly, the required execution time for microcontrollers is decreased. Based on this potentiality, future research work will be focused on improving the controller performance in advance for practical implementation.

\section{ACKNOWLEDGEMENT}

Our research is funded by the project of National University of Civil Engineering (NUCE) numbered 30-2021/KHXD-TĐ.

\section{REFERENCES}

[1] K. Dhineshkumar, C. Subramani, A. Geetha, and C. Vimala, "Performance analysis of PV powered multilevel inverter," International Journal of Electrical and Computer Engineering (IJECE), vol. 9, no. 2, p. 753-760, April 2019, doi: 10.11591/ijece.v9i2.pp753-760.

[2] P. Barbosa, P. Steimer, L. Meysenc, M. Winkelnkemper, J. Steinke, and N. Celanovic, "Active Neutral-PointClamped Multilevel Converters,” 2005 IEEE 36th Power Electronics Specialists Conference, 2005, pp. 2296-2301, doi: 10.1109/PESC.2005.1581952.

[3] S. Du, B. Wu, N. R. Zargari, and Z. Cheng, "A Flying-Capacitor Modular Multilevel Converter for MediumVoltage Motor Drive," IEEE Transactions on Power Electronics, vol. 32, no. 3, pp. 2081-2089, March 2017, doi: 10.1109/TPEL.2016.2565510. 
[4] M. V. Chung, D. T. Anh, P. Vu, and M. L. Nguyen, "Hardware in the loop co-simulation of finite set-model predictive control using fpga for a three level CHB inverter," International Journal of Power Electronics and Drive Systems (IJPEDS), vol. 11, no. 4, pp. 1719-1730, December 2020, doi: 10.11591/ijpeds.v11.i4.pp1719-1730.

[5] V. C. Mai, M. L. Nguyen, T. H. Vo, P. V. Hoang, and T. M. Tran, "Hardware In the Loop Simulation of Predictive Current Control for IM Fed by Multi-Level Cascaded H-Bridge Inverters," 2019 IEEE Vehicle Power and Propulsion Conference (VPPC), 2019, pp. 1-5, doi: 10.1109/VPPC46532.2019.8952288.

[6] C. M. Van, T. N. Xuan, P. V. Hoang, M. T. Trong, S. P. Cong, and L. N. Van, "A Generalized Space Vector Modulation for Cascaded H-bridge Multi-level Inverter," 2019 International Conference on System Science and Engineering (ICSSE), 2019, pp. 18-24, doi: 10.1109/ICSSE.2019.8823465.

[7] S. Wei, N. Zargari, B. Wu, and S. Rizzo, "Comparison and mitigation of common mode voltage in power converter topologies," Conference Record of the 2004 IEEE Industry Applications Conference, 2004. 39th IAS Annual Meeting., 2004, pp. 1852-1857 vol.3, doi: 10.1109/IAS.2004.1348722.

[8] Yen-Shin Lai, Po-Sheng Chen, Hsiang-Kuo Lee, and J. Chou, "Optimal common-mode voltage reduction PWM technique for inverter control with consideration of the dead-time effects-part II: applications to IM drives with diode front end," IEEE Transactions on Industry Applications, vol. 40, no. 6, pp. 1613-1620, Nov.-Dec. 2004, doi: 10.1109/TIA.2004.836151.

[9] I. Seo, N. B. Belaynehn, C. Park, and J. Kim, "A Study of Common Mode Voltage Generation According to Modulation Methods and Reduction Strategies on MMC System," 2018 IEEE Energy Conversion Congress and Exposition (ECCE), 2018, pp. 3988-3995, doi: 10.1109/ECCE.2018.8557920.

[10] J. Kalaiselvi and S. Srinivas, "Passive common mode filter for reducing shaft voltage, ground current, bearing current in dual two level inverter fed open end winding induction motor," 2014 International Conference on Optimization of Electrical and Electronic Equipment (OPTIM), 2014, pp. 595-600, doi: 10.1109/OPTIM.2014.6850977.

[11] H. Akagi, H. Hasegawa, and T. Doumoto, "Design and performance of a passive EMI filter for use with a voltagesource PWM inverter having sinusoidal output voltage and zero common-mode voltage," IEEE Transactions on Power Electronics, vol. 19, no. 4, pp. 1069-1076, July 2004, doi: 10.1109/TPEL.2004.830039.

[12] H. Truong, C. Mai, C. Nguyen, and P. Vu, "Modified Space Vector Modulation for Cascaded H-Bridge Multilevel Inverter with Open-Circuit Power Cells," Journal of Electrical and Computer Engineering, vol. 2021, pp. 1-14, 2021, doi: 10.1155/2021/6643589.

[13] L. G. G. P. de Castro, M. B. R. Corrêa, and C. B. Jacobina, "A fast space-vector algorithm for common-mode voltage elimination in multilevel converters," 2013 Brazilian Power Electronics Conference, 2013, pp. 243-247, doi: 10.1109/COBEP.2013.6785122.

[14] C. Mai-Van, S. Duong-Minh, D. Tran-Huu, B. Binh-Pho, and P. Vu, "An improved method of model predictive current control for multilevel cascaded H-bridge inverters," Journal of Electrical Engineering, vol. 72, no. 1, pp. 111, 2021, doi: 10.2478/jee-2021-0001.

[15] S. Kwak and S. Mun, "Model Predictive Control Methods to Reduce Common-Mode Voltage for Three-Phase Voltage Source Inverters," IEEE Transactions on Power Electronics, vol. 30, no. 9, pp. 5019-5035, Sept. 2015, doi: 10.1109/TPEL.2014.2362762.

[16] T. Geyer, P. Karamanakos, and R. Kennel, "On the benefit of long-horizon direct model predictive control for drives with LC filters," 2014 IEEE Energy Conversion Congress and Exposition (ECCE), 2014, pp. 3520-3527, doi: 10.1109/ECCE.2014.6953879.

[17] T. Geyer and D. E. Quevedo, "Performance of Multistep Finite Control Set Model Predictive Control for Power Electronics," IEEE Transactions on Power Electronics, vol. 30, no. 3, pp. 1633-1644, March 2015, doi: 10.1109/TPEL.2014.2316173.

[18] F. Grimm, P. Kolahian, Z. Zhang, and M. Baghdadi, "A Sphere Decoding Algorithm for Multistep Sequential Model-Predictive Control," IEEE Transactions on Industry Applications, vol. 57, no. 3, pp. 2931-2940, May-June 2021, doi: 10.1109/TIA.2021.3060694.

[19] T. Geyer and D. E. Quevedo, "Multistep Finite Control Set Model Predictive Control for Power Electronics," IEEE Transactions on Power Electronics, vol. 29, no. 12, pp. 6836-6846, Dec. 2014, doi: 10.1109/TPEL.2014.2306939.

[20] P.Karamanakos, T. Geyer, N. Oikonomou, F. D. Kieferndorf, and S. Manias, "Direct model predictive control: A review of strategies that achieve long prediction intervals for power electronics," IEEE Industrial Electronics Magazine. 2014, doi: 10.1109/MIE.2013.2290474.

[21] R. Baidya, R. P. Aguilera, P. Acuña, S. Vazquez and H. d. T. Mouton, "Multistep Model Predictive Control for Cascaded H-Bridge Inverters: Formulation and Analysis," in IEEE Transactions on Power Electronics, vol. 33, no. 1, pp. 876-886, Jan. 2018, doi: 10.1109/TPEL.2017.2670567.

[22] R. Baidya, et al., "Dealing with Suboptimality in Multistep Model Predictive Control for Transient Operations," 2019 IEEE Energy Conversion Congress and Exposition (ECCE), 2019, pp. 3780-3785, doi: 10.1109/ECCE.2019.8912815.

[23] F. Wang, et al., "An Encoderless Predictive Torque Control for an Induction Machine With a Revised Prediction Model and EFOSMO," IEEE Transactions on Industrial Electronics, vol. 61, no. 12, pp. 6635-6644, Dec. 2014, doi: 10.1109/TIE.2014.2317140.

[24] M. M. Bech, J. K. Pedersen, and F. Blaabjerg, "Field-oriented control of an induction motor using random pulsewidth modulation," in IEEE Transactions on Industry Applications, vol. 37, no. 6, pp. 1777-1785, Nov.-Dec. 2001, doi: 10.1109/28.968191. 
[25] A. K. Ali and R. G. Omar, "Finite control set model predictive direct current control strategy with constraints applying to drive three-phase induction motor," International Journal of Electrical and Computer Engineering (IJECE), vol. 11, no. 4, pp. 2916-2924, August 2021, doi: 10.11591/ijece.v11i4.pp2916-2924.

\section{BIOGRAPHIES OF AUTHORS}

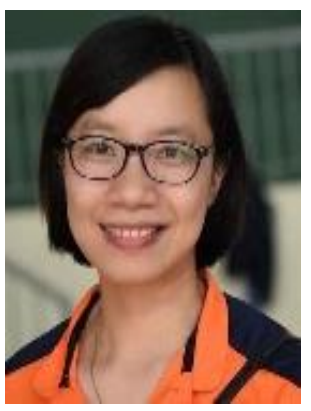

Pho Bao Binh received her MS degree from Hanoi University of Science and Technology, Vietnam in 2006 in Control Engineering and Automation. Since 2019, she has started to study her PhD degree at Hanoi University of Science and Technology. Her research interests include multilevel converter, model predictive current control.

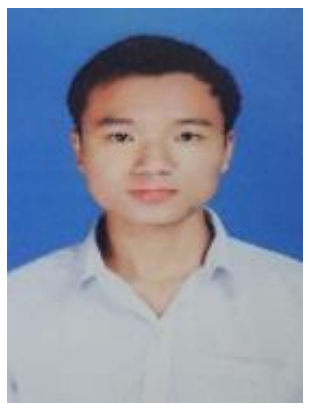

Nguyen Van Cao has been a student at School of Electrical Engineering, Hanoi University of Science and Technology, Vietnam since 2016. He is a member of Power Electronic Laboratory managed by PhD Phuong Vu-Hoang. His research interests include cascaded H-bridge multilevel converter and its applications in electrical machine drive and photovoltaic system.

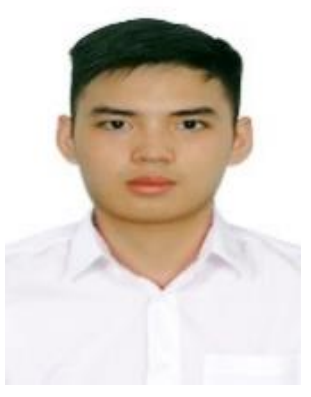

Tran Minh Hoan is a student at Hanoi University of Science and Technology. Since 2019, he has been a member of Power Electronic Laboratory managed by PhD Phuong Vu-Hoang. His current research interest are the high-power converters, especially the cascaded H-bridge multilevel inverter.

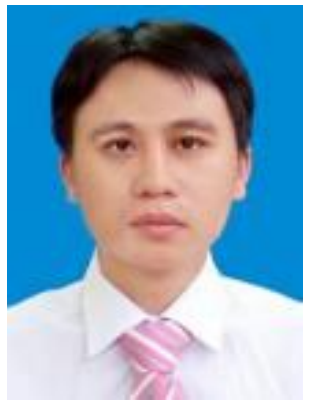

Phuong Vu received his B.S., M.S., and Ph.D. degrees from Hanoi University of Science and Technology, Vietnam, in 2006, 2008, and 2014, respectively, all in Control Engineering and Automation. Since 2006 he has been employed at Hanoi University of Science and Technology, where he is a lecturer and researcher at school of electrical engineering. His research interests include modeling and controlling of power electronics converters for applications such as photovoltaic, wind system, electrical machine drive. 